\section{Magnetic resonance imaging and pathological characteristics of pure mucinous carcinoma in the breast according to echogenicity on ultrasonography}

\author{
Young Gyung Shin, Eun-Kyung Kim, Min Jung Kim, Jung Hyun Yoon, Hee Jung Moon \\ Department of Radiology and Research Institute of Radiological Science, Severance Hospital, \\ Yonsei University College of Medicine, Seoul, Korea
}

Purpose: The aim of this study was to explore the clinical and pathological characteristics of pure mucinous breast carcinoma (PMBC) according to internal echogenicity on ultrasonography (US).

Methods: Thirty-three patients with PMBC diagnosed at surgery were included in this study. Cases of PMBC were classified according to internal echogenicity on US. The imaging features on magnetic resonance (MR) imaging and clinicohistopathological characteristics were compared between the hypoechogenic and the isoechogenic to hyperechogenic groups.

Results: Eleven cases of PMBC (33.3\%) exhibited hypoechogenicity on US, while 22 cases (66.7\%) exhibited isoechogenicity or hyperechogenicity. Of the isoechogenic to hyperechogenic PMBCs, 95.5\% showed a high signal on T2-weighted images, which was a significantly greater percentage than was observed for the hypoechogenic group (54.5\%) $(P=0.010)$. Of the hypoechogenic PMBCs, 63.6\% showed a washout pattern in the delayed phase, which was substantially more than the result of $23.8 \%$ observed for the isoechogenic to hyperechogenic PMBCs ( $P=0.053)$.

Conclusion: PMBCs with isoechogenicity or hyperechogenicity were more likely to show a high signal intensity on T2-weighted images than hypoechogenic PMBCs. However, other MR imaging and clinicohistopathological characteristics were not significantly different between the two groups.

Keywords: Breast neoplasms; Adenocarcinoma, mucinous; Magnetic resonance imaging; Ultrasonography

\section{Introduction}

Mucinous carcinoma of the breast is a rare subtype of invasive ductal carcinoma with an incidence of $1 \%-7 \%$, and it usually occurs in women over 60 years of age $[1,2]$. Mucinous carcinoma can be classified into two types: pure mucinous carcinoma, which has a mucinous component of more

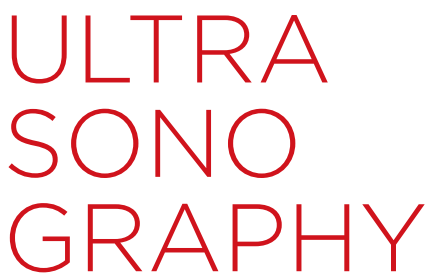

ORIGINAL ARTICLE

http://dx.doi.org/10.14366/usg.16028 pISSN: 2288-5919 e elSSN: 2288-5943 Ultrasonography 2017;36:131-138

Received: May 31, 2016

Revised: August 16, 2016

Accepted: August 29, 2016

Correspondence to: Hee Jung Moon, MD, PhD, Department of Radiology and Research Institute of Radiological Science, Severance Hospital, Yonsei University College of Medicine, 50-1 Yonsei-ro, Seodaemungu, Seoul 03722, Korea

Tel. $+82-2-2228-7400$

Fax. +82-2-393-3035

E-mail: artemis4u@yuhs.ac

This is an Open Access article distributed under the terms of the Creative Commons Attribution NonCommercial License (http://creativecommons.org/ licenses/by-nc/3.0/) which permits unrestricted noncommercial use, distribution, and reproduction in any medium, provided the original work is properly cited.

Copyright (C) 2017 Korean Society of Ultrasound in Medicine (KSUM)

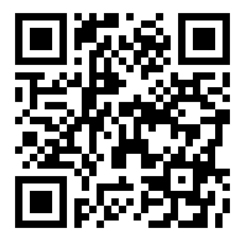

How to cite this article:

Shin YG, Kim EK, Kim MJ, Yoon JH, Moon HJ. Magnetic resonance imaging and pathological characteristics of pure mucinous carcinoma in the breast according to echogenicity on ultrasonography. Ultrasonography. 2017 Apr;36(2):131-138. 
than $90 \%$; and mixed mucinous carcinoma, which has a mucinous component greater than $50 \%$ but less than $90 \%$, admixed with areas with distinct architectural patterns, usually of invasive carcinoma of no special type $[3,4]$. Pure mucinous breast carcinoma (PMBC) is less aggressive and is less likely to lead to lymph node metastasis than mixed mucinous carcinoma $[1,5-7]$.

PMBC commonly presents as a well-circumscribed mass on mammography $[2,8]$. On ultrasonography (US), $64 \%-86 \%$ of PMBCs show isoechogenicity $[2,9,10]$. On magnetic resonance (MR) imaging, PMBC shows a high signal intensity on T2-weighted or short tau inversion recovery (STIR) T2-weighted images [11-14], and no restricted diffusion with high apparent diffusion coefficient (ADC) values on diffusion-weighted images $[14,15]$.

To our knowledge, no study has evaluated the clinical and pathological characteristics of PMBC according to echogenicity on US. Therefore, the demographic, MR imaging-related, and histologic characteristics of PMBC were investigated according to internal echogenicity on US.

\section{Materials and Methods}

The Institutional Review Board of our institution approved this retrospective study and waived the requirement for informed consent.

\section{Study Population}

From March 2012 to February 2015, 57 patients at our institution were diagnosed with mucinous carcinoma in the breast, of whom 48 had PMBC. Of these patients, the following were excluded: seven patients who underwent MR imaging after excision, five who underwent MR imaging using different protocols, two who underwent MR imaging at another hospital, and one patient who was male. Ultimately, 33 female patients who underwent breast MR imaging were included. The median age of the patients was 47 years (range, 31 to 79 years). The median lesion size was $17 \mathrm{~mm}$ (range, 5 to $37 \mathrm{~mm}$ ) on MR imaging.

\section{Mammography and US}

Mammography was performed using the Lorad/Hologic Selenia fullfield digital mammography system (Lorad/Hologic, Danbury, CT, USA) and the General Electric Senographe digital mammography system (GE Medical Systems, Milwaukee, WI, USA). Craniocaudal and mediolateral oblique views were routinely obtained. Additional views were obtained when needed. Breast density was classified according to the American College of Radiology Breast Imaging Reporting and Data System (ACR BI-RADS) $[16,17]$ and was recorded in the original radiological reports. Mammographic density was categorized as fatty (scores of A or B and 1 or 2 ) or dense (scores of $C$ or $D$ and 3 or 4$)$.

Breast US examinations were performed by 11 board-certified radiologists with 1 to 21 years of experience in breast imaging using US machines (iU22, Philips-Advanced Technology Laboratories, Bothell, WA, USA; Logic 9, GE Medical Systems) and 5-12- or 7-12-MHz linear array transducers. The echogenicity of the tumors on US was analyzed according to the ACR BI-RADS criteria $[16,17]$. The internal echo patterns of the tumors were retrospectively reanalyzed by one radiologist with 13 years of experience in breast imaging (H.J.M.). The tumors were classified into two categories according to echogenicity in comparison to the echogenicity of the premammary fat tissue, resulting in a hypoechogenic group and an isoechogenic to hyperechogenic group [17].

\section{MR Imaging Technique}

MR imaging of the breast was performed with a 3.0-T MR system (Discovery MR750w, GE Healthcare, Milwaukee, WI, USA). Commercially dedicated phased array breast coils were used in all cases with the patient in the prone position. Axial T2-weighted images were acquired using a fast spin-echo sequence (repetition time [TR]/echo time [TE], 4,187 msec/102 msec; field of view [FOV], $320 \times 320 \mathrm{~mm}$; matrix, $320 \times 256$ pixels; section thickness, $3 \mathrm{~mm}$ ), and axial STIR T2-weighted images (TR/TE, 5,000 msec/70 msec; inversion time, $200 \mathrm{msec}$ ) were acquired. After obtaining diffusionweighted MR images with a 2-dimensional spin-echo echo-planar imaging sequence, axial T1-weighted dynamic contrast-enhanced MR images of the entire breast were acquired before and 5 times after the intravenous administration of gadopentetate dimeglumine (Dotarem, Guerbet, Paris, France; Magnevist, Berlex Laboratories, Wayne, NJ, USA; Gaudiest, Bayer Schering Pharma, Berlin, Germany) using $0.2 \mathrm{~mL} / \mathrm{kg}$ at a rate of $2.0 \mathrm{~mL} / \mathrm{sec}$ (VIBRAT-Flex Din. imaging, GE Healthcare; matrix, 280×512 pixels; flip angle, 12\%; FOV, $320 \times 320 \mathrm{~mm}$; section thickness, $3 \mathrm{~mm}$, no intersection gap). Thus, six postcontrast images were acquired at approximately $0,63,126$, 189,252 , and 315 seconds after the intravenous administration of contrast, and temporal samplings of the center of $k$-space for the post-contrast series were obtained at approximately 32, 95, 158, 221,284 , and 347 seconds. Subtraction images were generated.

The signal intensity on T2-weighted MR images and STIR T2weighted images was visually classified as low, isointense, or high relative to the surrounding mammary parenchymal tissue. The degree of internal enhancement was classified as low, isoenhanced, or high, relative to the surrounding enhancing parenchymal tissues on the second subtraction images. Lesion size, dynamic enhancement pattern, peak enhancement, and ADC values were measured using a commercially available computer-aided evaluation 
system (CADstream, Confirma, Inc., Kirkland, WA, USA). Dynamic enhancement patterns were categorized as slow, medium, or rapid for the early phase and washout, persistent, or plateau for the delayed phase. To measure ADC values, the region of interest was manually drawn on the $A D C$ map ( $b$ value $=0,600 \mathrm{sec} / \mathrm{mm}^{2}$ ) 3 times. The mean $A D C$ value was calculated.

\section{Histopathologic Analysis}

Pathologic characteristics such as pathologic lesion size, infiltrative or expanding tumor margins, nuclear and histologic grades, the presence or absence of lymph node metastases, and lymphovascular invasion were compared between the two groups. The groups were also compared according to the presence of estrogen receptor (ER), progesterone receptor (PR), and human epidermal growth factor receptor 2 (HER2). ER and PR assays were considered positive if at least $1 \%$ of tumor nuclei were positive. HER2 positivity was defined as HER2 immunochemistry results of 3+ or HER2 gene amplification by silver-enhanced in situ hybridization analysis $[18,19]$. Ki-67 proliferation of $<14 \%$ was defined as negative and $\geq 14 \%$ as positive [20].

\section{Data and Statistical Analysis}

PMBCs were classified according to internal echogenicity on US, with the hypoechogenic group including PMBCs that exhibited hypoechogenicity and the isoechogenic to hyperechogenic group including PMBCs that exhibited isoechogenicity or hyperechogenicity.

Table 1. Imaging characteristics of mucinous carcinoma according to echogenicity on ultrasonography

\begin{tabular}{|c|c|c|c|c|}
\hline US echogenicity & Total & Hypoechogenic $(n=11)$ & $\begin{array}{l}\text { Isoechogenic to hyperechogenic } \\
\qquad(\mathrm{n}=22)\end{array}$ & P-value \\
\hline Age, median (range, yr) & $47(31-79)$ & $58(38-79)$ & $46(31-69)$ & 0.069 \\
\hline Symptom & & & & $>0.990$ \\
\hline Negative & $10(30.3)$ & $3(27.3)$ & $7(31.8)$ & \\
\hline Palpable & $23(69.7)$ & $8(72.7)$ & $15(68.2)$ & \\
\hline Mammographic density & & & & 0.121 \\
\hline$A, B$ & $9(27.3)$ & $5(45.5)$ & $4(18.2)$ & \\
\hline$C, D$ & $24(72.7)$ & $6(54.5)$ & $18(81.8)$ & \\
\hline \multicolumn{5}{|l|}{ MR imaging features } \\
\hline Lesion size, median (range, mm) & $17(5-37)$ & $16(8-34)$ & $18(5-37)$ & 0.849 \\
\hline Signal intensity on T2-weighted images & & & & 0.010 \\
\hline Isointense & $6(18.2)$ & $5(45.5)$ & $1(4.5)$ & \\
\hline High & $27(81.8)$ & $6(54.5)$ & $21(95.5)$ & \\
\hline Signal intensity on STIRT2-weighted images & & & & 0.097 \\
\hline Isointense & $4(12.1)$ & $3(27.3)$ & $1(4.5)$ & \\
\hline High & $29(87.9)$ & $8(72.7)$ & $21(95.5)$ & \\
\hline Internal enhancement & & & & 0.219 \\
\hline Low & $3(9.1)$ & 0 & $3(13.6)$ & \\
\hline Isoenhancement & $18(54.5)$ & $5(45.5)$ & $13(59.1)$ & \\
\hline High & $12(36.4)$ & $6(54.5)$ & $6(27.3)$ & \\
\hline Early enhancement & & & & $0.593^{b)}$ \\
\hline Medium & $4(12.5)$ & $2(18.2)$ & $2(9.5)$ & \\
\hline Rapid & $28(87.5)$ & $9(81.8)$ & $19(90.5)$ & \\
\hline Delay enhancement & & & & $0.053^{b)}$ \\
\hline Persistent or plateau & $20(62.5)$ & $4(36.4)$ & $16(76.2)$ & \\
\hline Washout & $12(37.5)$ & $7(63.6)$ & $5(23.8)$ & \\
\hline Peak enhancement, median (range, \%) & - & $203(84-376)$ & $215(66-549)$ & 0.166 \\
\hline ADC value, mean (range) & - & $1.90(1.59-1.95)$ & $1.92(1.17-2.44)$ & 0.133 \\
\hline
\end{tabular}

Values are presented as number (\%) unless otherwise indicated.

US, ultrasonography; MR, magnetic resonance; STIR, short tau inversion recovery; ADC, apparent diffusion coefficient.

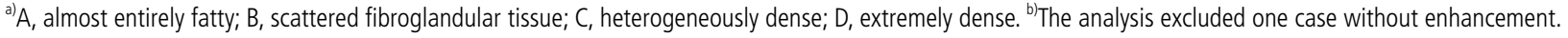


Patient age, the presence of symptoms, and mammographic density were compared between the two groups. Lesion size on MR imaging, signal intensity on T2-weighted images, signal intensity on STIR T2-weighted images, internal enhancement on the second contrast enhancement, early and delayed enhancement patterns, peak enhancement, and ADC values were also compared between the two groups.

The chi-square test and the Fisher exact test were used for categorical variables and the Mann-Whitney $U$ test was used for continuous variables. All results were analyzed using SPSS version 20.0 (IBM Corp., Armonk, NY, USA) and P-values of $<0.05$ were considered to indicate statistical significance.

\section{Results}

Of the 33 patients with PMBC, 11 (33.3\%) were classified into the hypoechogenic group and $22(66.7 \%)$ into the isoechogenic to hyperechogenic group. The median age of the patients in the hypoechogenic group was 58 years; this value was notably higher than the corresponding figure of 46 years in the isoechogenic to hyperechogenic group, although this difference was not statistically significant $(P=0.069)$ (Table 1). The presence of symptoms and

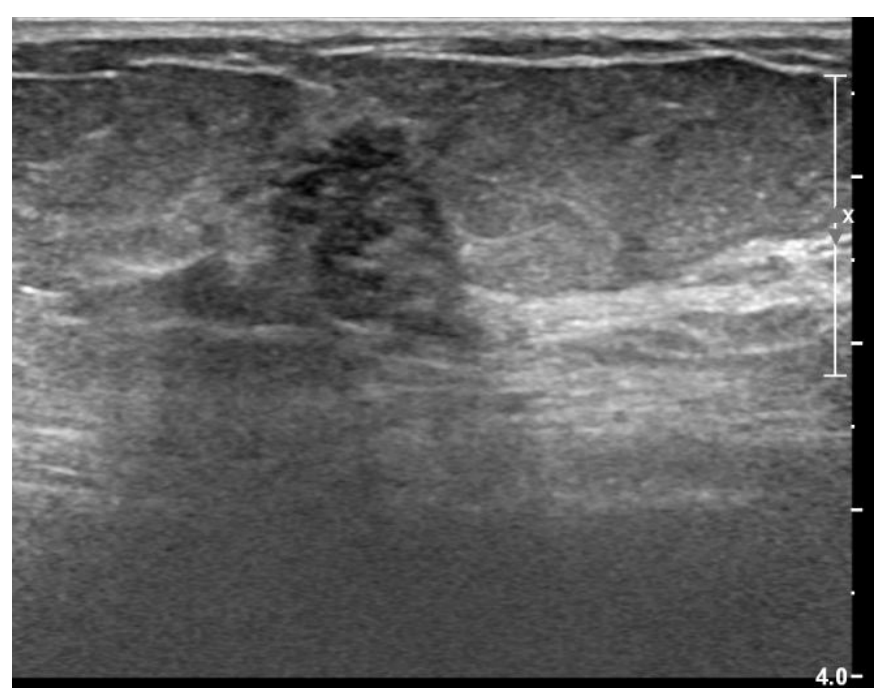

A

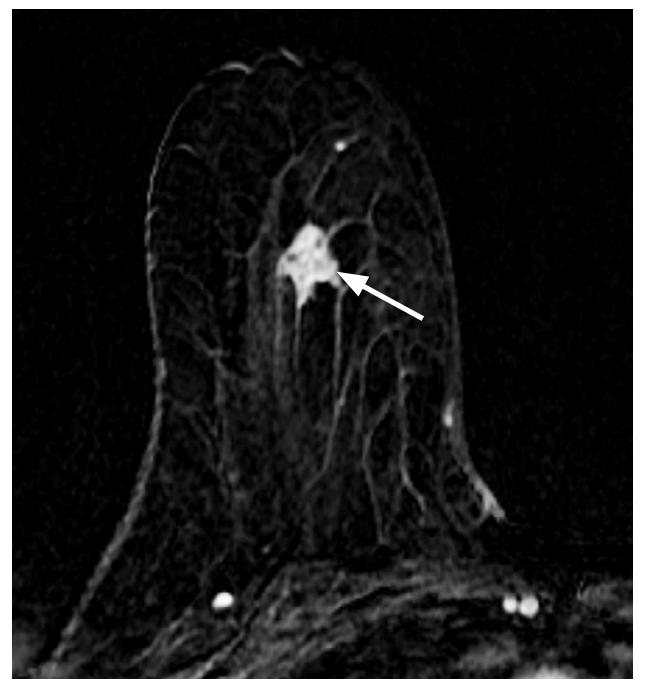

C

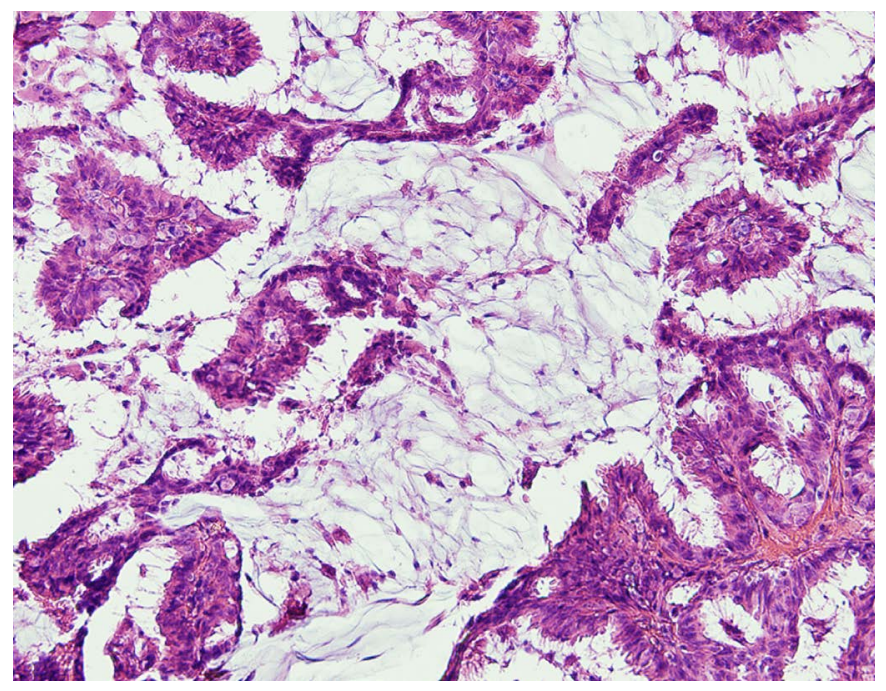

D

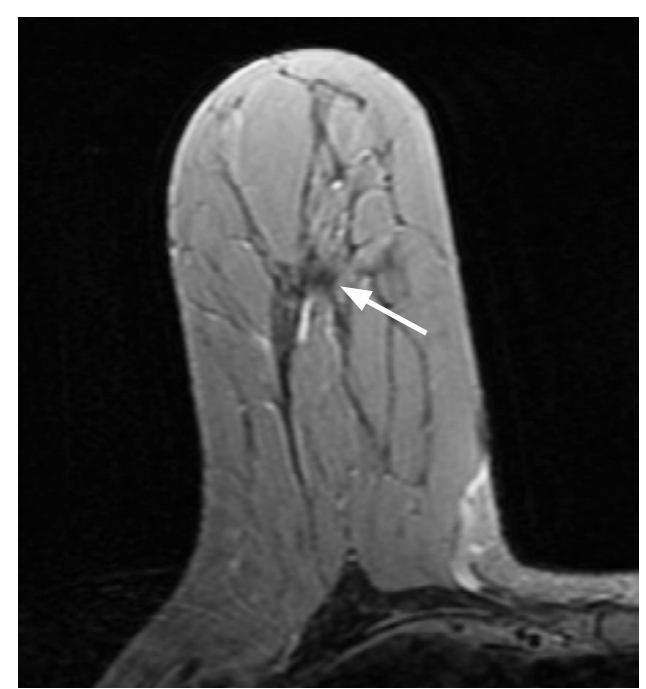

B

Fig. 1. A 75-year-old woman confirmed to have pure mucinous carcinoma of the right breast.

A. Ultrasonography of the area with a palpable lump in the right breast shows a 13-mm hypoechoic mass. B, C. The mass shows isointensity on T2-weighted images (arrow, B) and high internal enhancement on the second subtraction images (arrow, C). D. A low-power view visualizes the large lakes of extracellular mucin and clusters of tumor cells $(H \& E, \times 10)$. 
mammographic density were not significantly different between the two groups ( $P>0.990$ and $P=0.121$, respectively). Of $33 P M B C s$, $27(81.8 \%)$ and $29(87.9 \%)$ showed high signal intensity on T2weighted images and on STIR T2-weighted images, respectively. The PMBCs in the isoechogenic to hyperechogenic group were significantly more likely to show high signal intensity on T2weighted images (21 of 22,95.5\%) than the hypoechogenic group (six of $11,54.5 \%)(P=0.010)$ (Figs. 1, 2). On STIR T2-weighted images, $95.5 \%$ of the PMBCs in the isoechogenic to hyperechogenic group (21 of 22) showed a high signal intensity, in contrast to $72.7 \%$ in the hypoechogenic group (eight of 11), but this trend did not show statistical significance $(P=0.097)$. Internal enhancement, early and delayed enhancement patterns, peak enhancement, and mean $A D C$ values did not exhibit significant differences between the two groups $(P=0.219, P=0.593, P=0.053, P=0.166$, and $P=0.133$, respectively).

None of the 33 patients with PMBC had lymph node metastasis. Lesion size on pathology, tumor margin, nuclear and histologic grades, and lymphovascular invasion were not significantly different between the two groups ( $P=0.778, P>0.990, P=0.538, P=0.121$, and $P>0.990$ ) (Table 2). All mucinous carcinomas showed $E R$ positivity. PR positivity was not significantly different between the

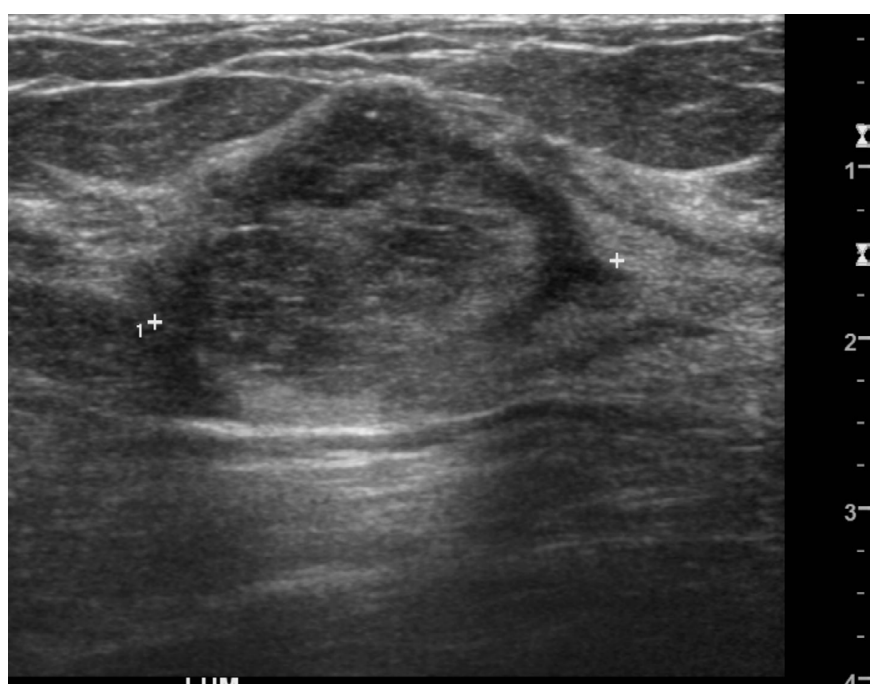

A

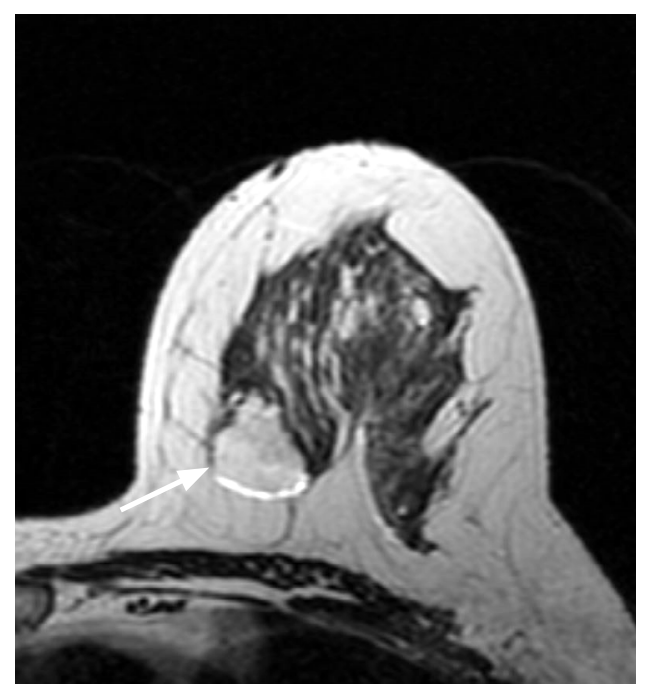

B

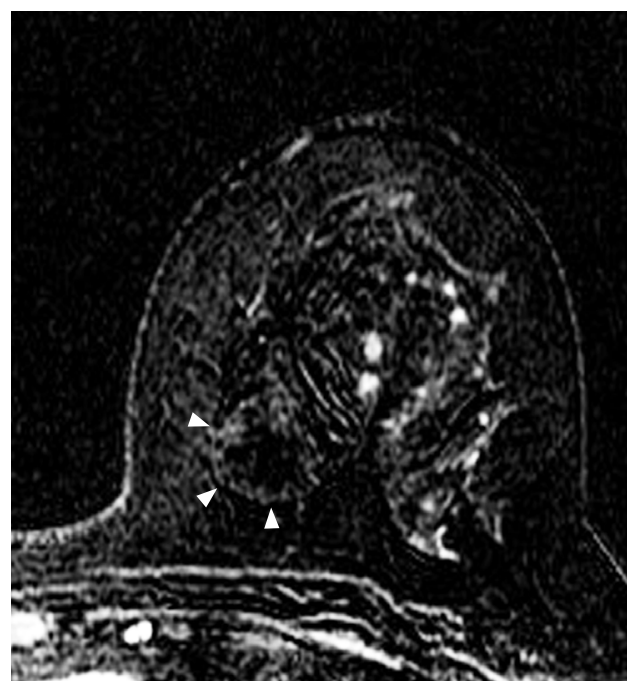

C

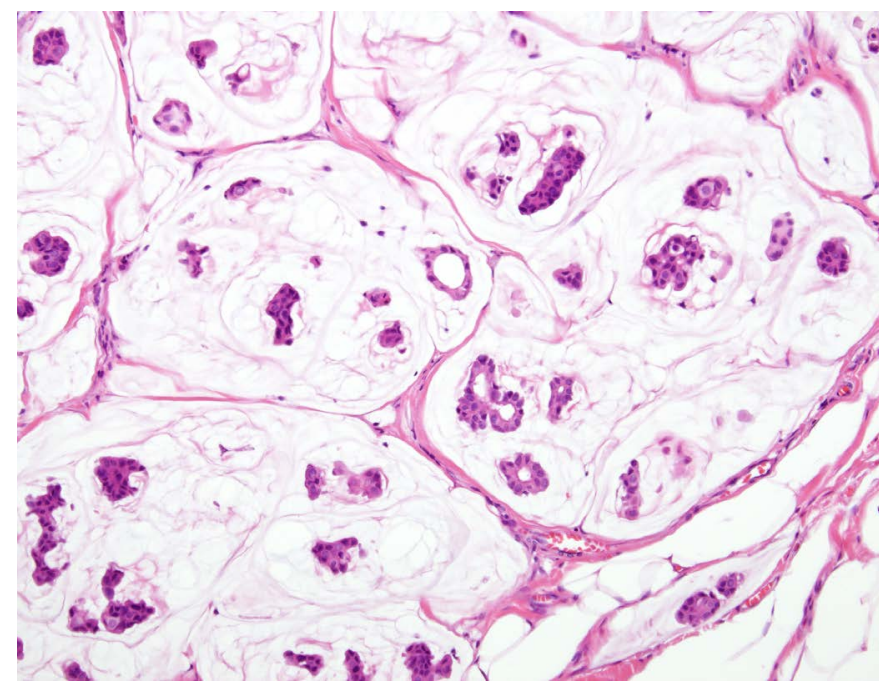

D

Fig. 2. A 44-year-old woman confirmed to have pure mucinous carcinoma of the left breast.

A. Ultrasonography of the area with a palpable lump in the left breast shows a 27-mm hyperechoic mass. B, C. The mass shows high signal intensity on T2-weighted images (arrow, B) and no internal enhancement on the second subtraction images (arrowheads, C). D. A low-power view visualizes small islands of tumor cells within lakes of extracellular mucin $(H \& E, \times 100)$. 
Table 2. Histopathologic characteristics of mucinous carcinoma according to echogenicity on US

\begin{tabular}{|c|c|c|c|c|}
\hline US echogenicity & Total & Hypoechogenic $(n=11)$ & Isoechogenic to hyperechogenic $(n=22)$ & P-value \\
\hline Lesion size $(\mathrm{mm})$ & $20(3-40)$ & $20(3-38)$ & $19(8-40)$ & 0.778 \\
\hline Margin & & & & $>0.990$ \\
\hline Expanding & $22(66.7)$ & $7(63.6)$ & $15(68.2)$ & \\
\hline Infiltrative & $11(33.3)$ & $4(36.4)$ & $7(31.8)$ & \\
\hline Nuclear grade & & & & 0.538 \\
\hline 1 & $16(48.5)$ & $4(36.4)$ & $12(54.5)$ & \\
\hline 2 & $17(51.5)$ & $7(63.6)$ & $10(45.5)$ & \\
\hline Histologic grade & & & & 0.121 \\
\hline 1 & $24(72.7)$ & $6(54.5)$ & $18(81.8)$ & \\
\hline 2 & $9(27.3)$ & $5(45.5)$ & $4(18.2)$ & \\
\hline \multicolumn{5}{|l|}{ Lymph node metastasis } \\
\hline Absence & $33(100)$ & $11(100)$ & $22(100)$ & \\
\hline Lymphovascular invasion & & & & $>0.990$ \\
\hline Absence & $31(93.9)$ & $10(90.9)$ & $21(95.5)$ & \\
\hline Presence & $2(6.1)$ & $1(9.1)$ & $1(4.5)$ & \\
\hline \multicolumn{5}{|l|}{ ER } \\
\hline Positive & $33(100)$ & $11(100)$ & $22(100)$ & \\
\hline$P R$ & & & & 0.304 \\
\hline Positive & 28 (84.8) & $8(72.7)$ & $20(90.9)$ & \\
\hline Negative & $5(15.2)$ & $3(27.3)$ & $2(9.1)$ & \\
\hline HER2 $2^{\text {a) }}$ & & & & 0.312 \\
\hline Positive & $1(3.1)$ & $1(10)$ & 0 & \\
\hline Negative & $31(96.9)$ & $9(90)$ & $22(100)$ & \\
\hline \multicolumn{5}{|l|}{$\mathrm{Ki}-67^{\mathrm{b})}$} \\
\hline Median (range) & $5(1-20)$ & $7(2-20)$ & $5(1-20)$ & 0.606 \\
\hline Positive ( $\geq 14 \%$ ) & $5(15.6)$ & $3(27.3)$ & $2(9.5)$ & 0.310 \\
\hline Negative (<14\%) & $27(84.4)$ & $8(72.7)$ & $19(90.5)$ & \\
\hline
\end{tabular}

Values are presented as median (range) or number (\%).

US, ultrasonography; ER, estrogen receptor; PR, progesterone receptor; HER2, human epidermal growth factor receptor 2.

${ }^{a}$ The analysis excluded one case with hypoechogenicity that had a 2+ HER2 immunohistochemistry result, but for which an additional silver-enhanced in situ hybridization analysis was not performed. ${ }^{\text {b) }}$ The analysis excluded one case with hyperechogenicity for which a Ki-67 assay was not performed.

two groups $(\mathrm{P}=0.304)$. Only one tumor was positive for HER2 in the hypoechogenic group and none were positive in the isoechogenic to hyperechogenic group $(P=0.312)$. No significant differences were observed between the two groups when the cut-off value of $\geq 14 \%$ was adopted for Ki-67 proliferation.

\section{Discussion}

PMBCs with isoechogenicity or hyperechogenicity were significantly more likely to show high signal intensity on T2-weighted images than PMBCs that exhibited hypoechogenicity. The histopathologic characteristics of PMBCs did not significantly differ according to their internal echogenicity on US.

In the hypoechogenic group and the isoechogenic to hyperechogenic group, $82 \%$ and $88 \%$ of PMBCs showed high signal intensity on T2- and STIR T2-weighted images in our study, respectively, which is comparable to previously reported findings of $82 \%$ to $100 \%$ for T2-weighted images $[12,13,21]$ and $67 \%$ to $100 \%$ for STIR T2-weighted images $[11,14]$. The large amount of extracellular mucus, which is rich in free water, in PMBCs is thought to contribute to their high signal intensity on T2-weighted and STIR T2-weighted MR images [11,12,21]. PMBCs with isoechogenicity or hyperechogenicity showed high signal intensity on T2-weighted images more frequently than those with hypoechogenicity. This 
is most likely because PMBCs with hypoechogenicity have more hypercellularity and less extracellular mucin. On STIR T2-weighted images, $72.7 \%$ of PMBCs in the hypoechogenic group showed a high signal intensity, in contrast to $54.5 \%$ of hypoechogenic PMBCs on T2-weighted images. STIR sequences increase the relative signal intensity of water content as a result of the additive T1 and T2 contrast effect, and this results in a notable contrast between water content and other tissues [22]. The increased contrast between mucin and the background parenchymal tissue might be the reason for this difference.

The mean $A D C$ values were not significantly different between the two groups $\left(1.90 \times 10^{-3} \mathrm{~mm}^{2} / \mathrm{sec}\right.$ vs. $\left.1.92 \times 10^{-3} \mathrm{~mm}^{2} / \mathrm{sec}, \mathrm{P}=0.133\right)$, similar to the ADC value of $1.99 \times 10^{-3} \mathrm{~mm}^{2} / \mathrm{sec}$ in a previous study [14]. This might be because the number of PMBCs included in our study was small, and the relative hypercellularity of PMBC with hypoechogenicity might not have been a strong enough trend to cause a significant difference in ADC values.

Our results showed that $62.5 \%$ of our sample of PMBCs exhibited persistent or plateau enhancement pattern in the delayed phase, while $37.5 \%$ showed a washout pattern. Delayed enhancement patterns were substantially different between the two groups. Of the PMBCs with hypoechogenicity, $63.6 \%$ showed a washout pattern on the delayed phase, substantially more than the corresponding value of $23.8 \%$ of PMBCs with isoechogenicity or hyperechogenicity. This difference in the delayed enhancement pattern probably occurred because the abundant mucin delays the diffusion of contrast medium throughout the stroma and around the epithelial component $[1,12]$. Internal enhancement was not significantly different between the two groups. Of the PMBCs, 54.5\% and 9.1\% showed isoenhancement and low internal enhancement, respectively, which is consistent with previous studies that found $58.8 \%$ of PMBCs to show mild enhancement and $5.9 \%$ to be without enhancement [13].

The pathological characteristics were not significantly different between the 2 groups. All PMBCs had nuclear and histologic grades of 1 or 2, corresponding to previous results showing $89 \%$ and $100 \%$ of PMBCs to be grade 1 or $2[1,23]$. None of the PMBCs exhibited lymph node metastasis, in contrast to previous studies that reported $0 \%-16.7 \%$ of PMBCs to have lymph node metastasis $[1,5,6,8,23-25]$. Our study found lymphovascular invasion in $6.1 \%$ of PMBCs, similar to the values of $5.7 \%-10.3 \%$ that have been reported in other studies $[23,24]$. PMBCs are slow-growing and are known to have a favorable prognosis due to the presence of mucin lakes, which are not vascularized and can slow tumor growth [1]. Although, hypocellular breast tumors have a more favorable prognosis [26], the relative hypercellularity of PMBCs in the hypoechogenic group did not lead to significant differences in the pathologic characteristics.

No statistically significant differences in immunohistochemical staining were found between the two groups. All PMBCs were positive for $E R$, and $84.8 \%$ were positive for $P R$, comparable with previously reported rates of $91 \%-100 \%$ for ER-positivity and $79 \%$ $85.7 \%$ for PR-positivity $[23,24,27]$. Only $3.1 \%$ of PMBCs showed HER2-positivity in the hypoechogenic group, which is comparable to previous reports of $2.9 \%-7.7 \%$ for HER2 expression $[23,24]$. Of the PMBCs, $84.4 \%$ were negative for Ki-67 expression when $<14 \%$ was chosen as the cut-off value, which is comparable to the results of a previous study in which most PMBCs showed low Ki-67 proliferation [24].

Our study had several limitations. First, this study had a retrospective design and a small number of cases were included. Selection bias may have been present. Second, we did not consider menstrual status, which can influence the signal intensity of the background breast parenchyma.

In conclusion, PMBCs with isoechogenicity or hyperechogenicity were more likely to have a high signal intensity on T2-weighted images than PMBCs that exhibited hypoechogenicity. However, the pathological characteristics were not significantly different between PMBCs exhibiting isoechogenicity or hyperechogenicity and those that displayed hypoechogenicity.

ORCID: Young Gyung Shin: http://orcid.org/0000-0001-7276-6230; Eun-Kyung Kim: http://orcid.org/0000-0002-3368-5013; Min Jung Kim: http://orcid.org/0000-00034949-1237; Jung Hyun Yoon: http://orcid.org/0000-0002-2100-3513; Hee Jung Moon: http://orcid.org/0000-0002-5643-5885

\section{Conflict of Interest}

No potential conflict of interest relevant to this article was reported.

\section{References}

1. Toikkanen $\mathrm{S}$, Kujari H. Pure and mixed mucinous carcinomas of the breast: a clinicopathologic analysis of 61 cases with long-term follow-up. Hum Pathol 1989;20:758-764.

2. Memis A, Ozdemir N, Parildar M, Ustun EE, Erhan Y. Mucinous (colloid) breast cancer: mammographic and US features with histologic correlation. Eur J Radiol 2000;35:39-43.

3. Lakhani SR, Ellis. IO, Schnitt SJ, Tan PH, van de Vijver MJ. WHO classification of tumours of the breast. Vol. 4. 4th ed. Lyon: IARC Press, 2014.

4. Tan PH, Tse GM, Bay BH. Mucinous breast lesions: diagnostic challenges. J Clin Pathol 2008;61:11-19.

5. Di Saverio S, Gutierrez J, Avisar E. A retrospective review with long term follow up of 11,400 cases of pure mucinous breast carcinoma. Breast Cancer Res Treat 2008;111:541-547. 
6. Fentiman IS, Millis RR, Smith P, Ellul JP, Lampejo O. Mucoid breast carcinomas: histology and prognosis. Br J Cancer 1997;75:10611065.

7. Lam WW, Chu WC, Tse GM, Ma TK. Sonographic appearance of mucinous carcinoma of the breast. AJR Am J Roentgenol 2004;182:1069-1074.

8. Wilson TE, Helvie MA, Oberman HA, Joynt LK. Pure and mixed mucinous carcinoma of the breast: pathologic basis for differences in mammographic appearance. AJR Am J Roentgenol 1995; 165:285-289.

9. Chopra S, Evans AJ, Pinder SE, Yeoman LJ, Ellis IO, Elston CW, et al. Pure mucinous breast cancer-mammographic and ultrasound findings. Clin Radiol 1996;51:421-424.

10. Kaoku S, Konishi E, Fujimoto Y, Tohno E, Shiina T, Kondo K, et al. Sonographic and pathologic image analysis of pure mucinous carcinoma of the breast. Ultrasound Med Biol 2013;39:1158-1167.

11. Okafuji T, Yabuuchi H, Sakai S, Soeda H, Matsuo Y, Inoue T, et al. MR imaging features of pure mucinous carcinoma of the breast. Eur J Radiol 2006;60:405-413.

12. Kawashima M, Tamaki Y, Nonaka T, Higuchi K, Kimura M, Koida T, et al. MR imaging of mucinous carcinoma of the breast. AJR Am J Roentgenol 2002;179:179-183.

13. Monzawa S, Yokokawa M, Sakuma T, Takao S, Hirokaga K, Hanioka $\mathrm{K}$, et al. Mucinous carcinoma of the breast: MRI features of pure and mixed forms with histopathologic correlation. AJR Am J Roentgenol 2009;192:W125-W131.

14. Zhang L, Jia N, Han L, Yang L, Xu W, Chen W. Comparative analysis of imaging and pathology features of mucinous carcinoma of the breast. Clin Breast Cancer 2015;15:e147-e154.

15. Woodhams R, Kakita S, Hata H, Iwabuchi K, Umeoka S, Mountford $C E$, et al. Diffusion-weighted imaging of mucinous carcinoma of the breast: evaluation of apparent diffusion coefficient and signal intensity in correlation with histologic findings. AJR Am J Roentgenol 2009;193:260-266.

16. American College of Radiology. Breast Imaging Reporting and Data System (BI-RADS). 4th ed. Reston, VA: American College of Radiology, 2003.

17. D'Orsi CJ, Sickles EA, Mendelson EB, Morris EA. ACR BI-RADS Atlas, Breast Imaging Reporting and Data System. Reston, VA: American
College of Radiology, 2013.

18. Hammond ME, Hayes DF, Dowsett M, Allred DC, Hagerty KL, Badve $S$, et al. American Society of Clinical Oncology/College Of American Pathologists guideline recommendations for immunohistochemical testing of estrogen and progesterone receptors in breast cancer. J Clin Oncol 2010;28:2784-2795.

19. Wolff AC, Hammond ME, Schwartz JN, Hagerty KL, Allred DC, Cote RJ, et al. American Society of Clinical Oncology/College of American Pathologists guideline recommendations for human epidermal growth factor receptor 2 testing in breast cancer. J Clin Oncol 2007;25:118-145.

20. Cheang MC, Chia SK, Voduc D, Gao D, Leung S, Snider J, et al. Ki67 index, HER2 status, and prognosis of patients with luminal $B$ breast cancer. J Natl Cancer Inst 2009;101:736-750.

21. Yuen S, Uematsu T, Kasami M, Tanaka K, Kimura K, Sanuki J, et al. Breast carcinomas with strong high-signal intensity on T2-weighted MR images: pathological characteristics and differential diagnosis. J Magn Reson Imaging 2007;25:502-510.

22. Kijowski R, Farber JM, Medina J, Morrison W, Ying J, Buckwalter K. Comparison of fat-suppressed T2-weighted fast spin-echo sequence and modified STIR sequence in the evaluation of the rotator cuff tendon. AJR Am J Roentgenol 2005; 185:371-378.

23. Tseng HS, Lin C, Chan SE, Chien SY, Kuo SJ, Chen ST, et al. Pure mucinous carcinoma of the breast: clinicopathologic characteristics and long-term outcome among Taiwanese women. World J Surg Oncol 2013;11:139.

24. Lacroix-Triki M, Suarez PH, MacKay A, Lambros MB, Natrajan R, Savage $K$, et al. Mucinous carcinoma of the breast is genomically distinct from invasive ductal carcinomas of no special type. J Pathol 2010;222:282-298.

25. Avisar E, Khan MA, Axelrod D, Oza K. Pure mucinous carcinoma of the breast: a clinicopathologic correlation study. Ann Surg Oncol 1998;5:447-451.

26. Benson JR, Gui GP, Tuttle T. Early breast cancer: from screening to multidisciplinary management. 3rd ed. Boca Raton, FL: CRC Press, 2013.

27. Komenaka IK, El-Tamer MB, Troxel A, Hamele-Bena D, Joseph KA, Horowitz $E$, et al. Pure mucinous carcinoma of the breast. Am J Surg 2004; 187:528-532. 